\title{
ANUÁRIO DE LITERATURA
}

Volume 17

Número 2

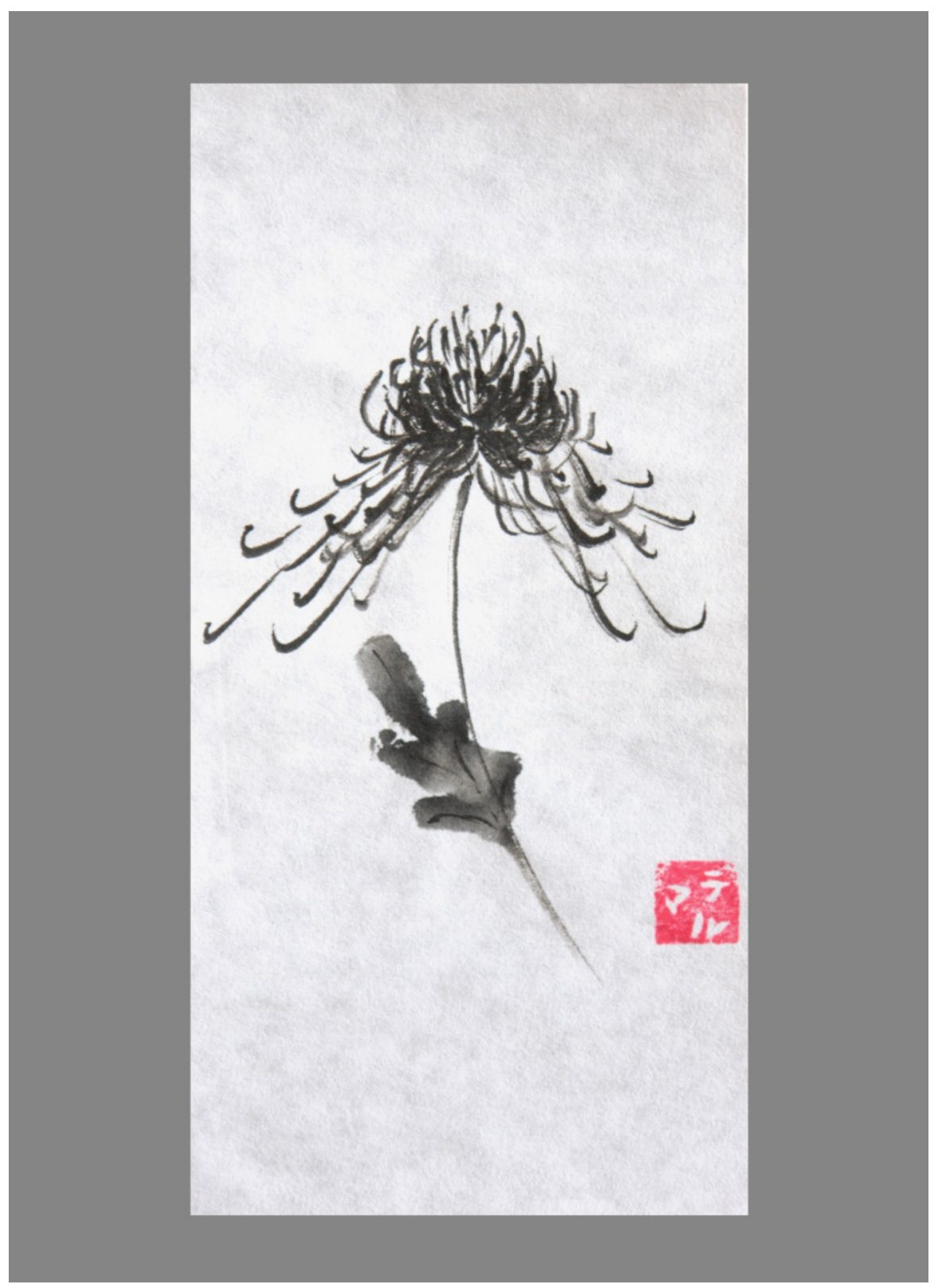

PROGRAMA DE PÓS-GRADUAÇÃO EM LITERATURA UNIVERSIDADE FEDERAL DE SANTA CATARINA

Dezembro de 2012, Florianópolis, Santa Catarina, Brasil 
Programa de Pós-Graduação em Literatura

Centro de Comunicação e Expressão

Universidade Federal de Santa Catarina

Campus Universitário - Trindade

88040-900 - Florianópolis - SC

Capa

Outono Kiko, Telma Piacentini

Layout

Andréa Figueiredo Leão Grants

Jair Zandoná

Editor-chefe

Gizelle Kaminski Corso

Stélio Furlan

Comissão Editorial

Andréa Figueiredo Leão Grants

Gizelle Kaminski Corso

Jair Zandoná

Stélio Furlan

Tanay Gonçalves Notargiacomo

\section{Conselho Consultivo}

Álvaro Santos Simões Jr., Universidade Estadual Paulista, Brasil

Ana Cecilia Acioli Lima, Universidade Federal de Alagoas, Brasil

Ana Cecília Olmos, Universidade de São Paulo, Brasil

Andrea do Roccio Souto, Universidade Federal de Santa Maria, Brasil

Andréia Guerini, Universidade Federal de Santa Catarina, Brasil

Annabela Rita, Universidade de Lisboa, Portugal

Artur Emílio Alarcon Vaz, Universidade Federal de Rio Grande, Brasil

Benedito Antunes, Universidade Estadual Paulista, Brasil

Carlos Eduardo Schmidt Capela, Universidade Federal de Santa Catarina, Brasil

Claudia Consuelo Amigo Pino, Universidade de São Paulo, Brasil

Clélia Maria Lima de Mello e Campigotto, Universidade Federal de Santa Catarina, Brasil

Cristiane Maria da Silva, Universidade Federal de Santa Catarina, Brasil

Daniela Bunn, Universidade Federal de Santa Catarina, Brasil

Dilma Beatriz Rocha Juliano, Universidade do Sul de Santa Catarina, Brasil

Edimilson de Almeida Pereira, Universidade Federal de Juiz de Fora, Brasil

Eliane Santana Dias Debus, Universidade Federal de Santa Catarina, Brasil

Fernanda Müller, Colégio de Aplicação, Universidade Federal de Santa Catarina, Brasil

Gilberto Figueiredo Martins, Universidade Estadual Paulista, Brasil

Giorgio de Marchis, Università degli Studi Roma Tre, Itália

Gladys Viviana Gelado, Universidade Federal Fluminense, Brasil

Helena Heloisa Fava Tornquist, Universidade Federal de Santa Catarina, Brasil

Homero José Vizeu de Araújo, Universidade Federal do Rio Grande do Sul, Brasil

Jade Gandra Dutra Martins, Universidade Federal de Santa Catarina, Brasil

Jair Tadeu da Fonseca, Universidade Federal de Santa Catarina, Brasil

João Hernesto Weber, Universidade Federal de Santa Catarina, Brasil 
Mauro Eduardo Pommer, Universidade Federal de Santa Catarina, Brasil

Marcio Markendorf, Universidade Federal de Santa Catarina, Brasil

Maria Adélia Menegazzo, Universidade Federal de Mato Grosso do Sul, Brasil

Mauri Furlan, Universidade Federal de Santa Catarina, Brasil

Nilcéia Valdati, Faculdades ASSESC, Brasil

Patricia Peterle, Universidade Federal de Santa Catarina, Brasil

Pedro de Souza, Universidade Federal de Santa Catarina, Brasil

Rafael Zamperetti Copetti, Universidade Federal de Santa Catarina, Brasil

Renata Praça de Souza Telles, Universidade Federal do Paraná, Brasil

Renato Cordeiro Gomes, Pontifícia Universidade Católica do Rio de Janeiro, Brasil

Rita Lenira de Freitas Bittencourt, Universidade Federal do Rio Grande do Sul, Brasil

Rita Marnoto, Universidade de Coimbra, Portugal

Rosana de Cássia Kamita, Universidade Federal de Santa Catarina, Brasil

Sérgio Luiz Rodrigues Medeiros, Universidade Federal de Santa Catarina, Brasil

Sergio Nazar David, Universidade do Estado do Rio de Janeiro, Brasil

Silvio Renato Jorge, Universidade Federal do Rio de Janeiro, Brasil

Susan Aparecida de Oliveira, Universidade Federal de Santa Catarina, Brasil

Tânia Celestino Macedo, Universidade de São Paulo, Brasil

Tania Regina Oliveira Ramos, Universidade Federal de Santa Catarina, Brasil

Tereza Virginia de Almeida, Universidade Federal de Santa Catarina, Brasil

Veridiana Almeida, Faculdade Educacional da Lapa, Brasil

Walter Carlos Costa, Universidade Federal de Santa Catarina, Brasil

Zahidé Lupinacci Muzart, Universidade Federal de Santa Catarina, Brasil

\section{Apoio Técnico}

Úrsula Blattmann (Biblioteconomia/UFSC)

Andréa Figueiredo Leão Grants (Bibliotecária/UFSC)

\section{Coordenadora do Programa de Pós-Graduação em Literatura}

Profa. Dra. Susana Scramim

Catalogação na fonte pela Biblioteca Universitária

da

Universidade Federal de Santa Catarina

Anuário de literatura [recurso eletrônico] / Programa de PósGraduação em Literatura. - Florianópolis, Universidade Federal de Santa Catarina. Centro de Comunicação e Expressão, 1993-

Vol. 17, n. 2

Semestral

ISSNe 2175-7917

Acesso em: http://www.periodicos.ufsc.br/index.php/literatura

1. Literatura brasileira. 2. Teoria literária. I. Universidade

Federal de Santa Catarina. Centro de Comunicação e Expressão.

CDU: 82 


\section{Anuário de Literatura}

anuariodeliteratura.cce@,contato.ufsc.br

Os textos publicados neste número foram revisados por seus autores e são de sua inteira responsabilidade. 